\title{
Analysis of imaging characteristics of primary malignant bone tumors in children
}

\author{
YINGWEI SUN $^{1 *}$, XUEYONG LIU ${ }^{2 *}$, SHINONG PAN $^{1}, \mathrm{CHUNBO} \mathrm{DENG}^{3}$, XIAOHAN LI ${ }^{4}$ and QIYONG GUO ${ }^{1}$ \\ Departments of ${ }^{1}$ Radiology and ${ }^{2}$ Orthopedics, Shengjing Hospital, China Medical University, Shenyang, \\ Liaoning 110004; ${ }^{3}$ Department of Orthopedics, Central Hospital, Shenyang Medical College, Shenyang, Liaoning 110024; \\ ${ }^{4}$ Department of Pathology, Shengjing Hospital, China Medical University, Shenyang, Liaoning 110004, P.R. China
}

Received March 22, 2016; Accepted July 11, 2017

DOI: $10.3892 / \mathrm{ol} .2017 .6939$

\begin{abstract}
The present study aimed to investigate the imaging characteristics of primary malignant bone tumors in children. The imaging results of 34 children with primary malignant bone tumors confirmed by histopathological diagnosis between March 2008 and January 2014 were retrospectively analyzed. In total, 25 patients had osteosarcoma, with radiography and computed tomography (CT) showing osteolytic bone destruction or/and osteoblastic bone sclerosis, an aggressive periosteal reaction, a soft-tissue mass and cancerous bone. The tumors appeared as mixed magnetic resonance imaging (MRI) signals that were inhomogeneously enhanced. A total of 5 patients presented with Ewing sarcoma, with radiography and CT showing invasive bone destruction and a soft-tissue mass. Of the 5 cases, 2 showed a laminar periosteal reaction. The tumors were shown to have mixed low signal on T1-weighted images (T1WI) and high signal on T2-weighted images (T2WI); 1 case showed marked inhomogeneous enhancement. Another 3 patients exhibited chondrosarcoma. Of these cases, 1 was adjacent to the cortex of the proximal tibia, and presented with local cortical bone destruction and a soft-tissue mass containing scattered punctate and amorphous calcifications. MRI revealed mixed low T1 signal and high T2 signals. Another case was located in the medullary cavity of the distal femur, with radiography revealing a localized periosteal reaction. The tumor appeared with mixed MRI signals, and with involvement of the epiphysis and epiphyseal plates. Radiography and CT of the third case showed bone destruction in the right pubic ramus, with patchy punctate, cambered calcifications in the soft-tissue mass. MRI of the soft-tissue mass revealed isointensity on T1WI and heterogeneous
\end{abstract}

Correspondence to: Professor Qiyong Guo, Department of Radiology, Shengjing Hospital, China Medical University, 36 Sanhao Street, Heping, Shenyang, Liaoning 110004, P.R. China E-mail: guoqysj@sina.com

${ }^{*}$ Contributed equally

Key words: bone tumor, malignant, children, imaging hyperintensity on T2WI. Ossifications and the septum appeared as low T1WI and T2WI. Of the 34 patients, 1 patient presented with lymphoma involving the T12, L1 and L2 vertebrae. CT showed vertebral bone destruction, a soft-tissue mass and a compression fracture of L1. MRI showed a soft-tissue mass with low T1 signal and high T2 signal and marked inhomogeneous enhancement. Overall, osteosarcoma was the most common primary malignant bone tumor, followed by Ewing sarcoma, chondrosarcoma and lymphoma. Osteoblastic or osteolytic bone destruction, an invasive periosteal reaction, soft-tissue masses, a tumor matrix and inhomogeneous enhancement were important imaging features of malignant bone tumors.

\section{Introduction}

The prevalence of primary malignant bone tumors is markedly lower than the prevalence of benign bone tumors in children, with malignant bone tumors accounting for only $6 \%$ of all bone tumors (1). Over $90 \%$ of cases of primary malignant bone tumors are osteosarcomas or Ewing sarcomas (2). Owing to their rapid and invasive growth, malignant bone tumors are a major cause of mortality and disability in children, despite their low incidence (3). Compared with the elderly and young adults, children and adolescents with malignant bone tumors have a lower survival rate and poorer prognosis; as such, malignant bone tumors in children are the focus of research (3).

Early diagnosis and treatment are important for improving the quality of life and survival rate of children with malignant bone tumors. If the tumor is diagnosed and treated early, limb salvage can be achieved during the tumor is resected. Motor function can also be reconstructed to improve quality of life and extend survival time $(4,5)$. However, diagnosis is frequently delayed for weeks to months, partly because the rarity of such tumors and that the presence of a malignancy in an otherwise healthy adolescent is unexpected (6). Therefore, performing an accurate diagnosis of malignant bone tumors in children is extremely important.

Radiography is the prime imaging modality for evaluation of primary bone tumors (7). The role of cross-sectional imaging, including magnetic resonance imaging (MRI), computed tomography (CT), and nuclear medicine (NM) technetium bone scan, plays an integral part in characteristics of the extent 
of tumors (8). In addition, dynamic MRI, diffusion weighted MRI and ${ }^{18} \mathrm{~F}$-fluorodeoxyglucose positron emission tomography-computed tomography $\left({ }^{18} \mathrm{FDG}-\mathrm{PET} / \mathrm{CT}\right)$ to monitor tumor necrosis $(1,8)$. These imaging modalities revolutionized diagnostic and therapeutic approaches to musculoskeletal oncology, which has a major role in evaluating metastases, guiding surgery and radiation and detecting response to treatment and tumor recurrence (5).

In the present study, 34 cases of pathologically confirmed primary malignant bone tumors in children were retrospectively analyzed. The aim of the present study was to investigate the imaging characteristics of primary malignant bone tumors in children.

\section{Patients and methods}

Patients. The study procedures were approved by the Ethics Committee of Shengjing Hospital (China Medical University, Shenyang, Liaoning, China) and were conducted in accordance with the Declaration of Helsinki. Patient records were anonymized and de-identified prior to analysis. The imaging results of 34 children, aged $<18$ years, who were diagnosed with a primary malignant bone tumor that was confirmed by histopathological diagnosis in Shengjing Hospital (China Medical University, Shenyang, Liaoning, China) between March 2008 to January 2014 were collected. Of the 34 patients, 18 were male and 16 were female, with a mean age of 10.8 years (range, 4-17 years). A total of 25 patients presented with osteosarcoma, 5 with Ewing sarcoma, 3 with chondrosarcoma and 1 with lymphoma. The main clinical symptoms included pain, swelling, gradually enlarging soft-tissue mass and pathological fractures.

Imaging. Anterior-posterior and lateral plain radiographs were performed using a digital radiography system (Kodak DirectView DR7500; Kodak, Rochester, NY, USA). Axial CT was performed using multislice CT scanners (Somatom Sensation 64/Definition 64; both Siemens Medical Solutions, Forchheim, Germany; and Brilliance 64; Philips Healthcare, Cleveland, $\mathrm{OH}, \mathrm{USA}$ ). The coronal and sagittal images were subject to multiplanar reconstruction. The scanning parameters were as follows: Tube voltage, $120 \mathrm{kV}$; tube current, $200 \mathrm{~mA}$; and slice thickness, $1 \mathrm{~mm}$. Non-ionic iodinated contrast agent $(80-100 \mathrm{ml})$ injected intravenously at a rate of $3.0 \mathrm{ml} / \mathrm{sec}$, was used for contrast enhancement. Whole-body bone scintigraphy was performed using a single-photon emission computed tomography imaging system (Infinia Hawkeye; GE Healthcare, Chicago, IL, USA). At $3 \mathrm{~h}$ after the intravenously administration of $725 \mathrm{MBq}(25 \mathrm{mCi})$ of Technetium-99 m methylene diphosphonate (99m Tc-MDP), anterior and posterior views of whole-body bone scintigraphy was obtained. ${ }^{18} \mathrm{~F}-\mathrm{FDG} \mathrm{PET} / \mathrm{CT}$ was performed using a PET/CT imaging system (Discovery ST, GE Healthcare, Chicago, IL, USA). ${ }^{18} \mathrm{~F}$-FDG was intravenously administered at a dose of $3.7 \mathrm{MBq} / \mathrm{kg}$. MRI was performed using superconducting MR systems (Signa HDxt3.0T; GE Healthcare; or Achieva 3.0T/Gyroscan Intera 1.5T; Philips Healthcare, Best, the Netherlands). A combination of axial, sagittal, and coronal images were obtained using T1-weighted spin-echo (SE) sequence, T2-weighted fast spin-echo (FSE) sequence, and
T2-weighted fat suppression sequences, including short-time inversion recovery (STIR), spectral-presaturation inversion recovery (SPIR), and iterative Dixon water-fat separation with echo asymmetry and least-squares estimation (IDEAL). The following acquisition parameters were employed: Slice thickness, 3.5-5 mm; field of view, 300-400 mm; and matrix, $512 \times 512$. A total of $0.1 \mathrm{mmol} / \mathrm{kg}$ gadolinium-diethylene triamine pentacetate acid, infused intravenously at $2 \mathrm{ml} / \mathrm{sec}$, was used for enhancement.

Imaging features of the tumors, including location, size, boundary, number and tumor matrix, type of bony destruction, soft-tissue mass and aggressive periosteal reaction, among others, were independently evaluated by two radiologists with 9 and 20 years of bone tumor MRI experience, respectively). If the judgment was inconsistent, the result was determined by a consensus after discussion.

Histology. All histological samples were reviewed by pathologist. All specimens were fixed in $10 \%$ formalin for $4 \mathrm{~h}$ at room temperature, embedded in paraffin, sectioned at a thickness of $5 \mu \mathrm{m}$ for hematoxylin-eosin (HE) staining and $3 \mu \mathrm{m}$ for immunohistochemical staining, followed by observed under light microscope.

Immunohistochemical staining performed with primary monoclonal antibodies targeted at: B-cell lymphoma 2, cluster of differentiation 99 (CD99), S-100, vimentin, LCA, neuron-specifc enolase, ALK tyrosine kinase receptor, CD3, CD10, CD20, CD30, CD68, CD117, epithelial membrane antigen, myeloperoxidase and T-cell intracellular antigen-1 (cat. nos. ZM-0010, ZM-0296, ZM-0224, ZM-0260, ZM-0183, ZM-0203, ZM-0248, ZA-0503, ZA-0526, ZM-0039, ZA-0591, ZM-0060, ZA-0523, ZA-0197 and ZM-0457, respectively; ready-to-use; Origene Technologies, Inc., Beijing, China).

The Bond Polymer Refine Detection kit (cat. no. DS9800; Leica Microsystems, Inc., Buffalo Grove, IL, USA) was applied using the primary monoclonal antibody. The incubation time for the primary antibody was $15 \mathrm{~min}$. The incubation time for the horseradish peroxidase conjugates was $8 \mathrm{~min}$ at $37^{\circ} \mathrm{C}$, and diaminobenzidine (DAB) incubation time was $10 \mathrm{~min}$. The procedure was performed by a professional pathologist using the Bond-Max autostainer (Leica Microsystems, Inc.) according to the manufacturer's instructions.

\section{Results}

The specific imaging characteristics of the 34 pediatric cases of malignant bone tumors are listed in the subsections that follow.

Osteosarcoma. There were 25 cases of osteosarcoma (12 males and 13 females), with patient ages ranging from 4 to 17 years, which were all located in the long bones, including 14 involving the distal femur, 7 in the proximal tibia and 4 in the proximal humerus. The types of bone destruction included 4 osteolytic, 4 osteoblastic and 17 mixed cases. The histopathology included 24 conventional osteosarcoma cases and 1 telangiectatic osteosarcoma case. A pathological fracture was present in 4 cases, skip lesions in 2 cases and lung metastasis in 5 cases. 
X-ray (22/25) and CT (21/25) revealed osteolytic and/or osteoblastic bone destruction. The size of the tumors ranged from $6.1-15.2 \mathrm{~cm}$. A total of 24 cases had fluffy, sunburst, spiculated and other aggressive periosteal reactions; of which 19 showed a Codman triangle. Of the 23 cases with a soft-tissue mass, 21 had punctate or irregular cancerous bone in the area of bone destruction and in the soft-tissue mass. A single case with lesions in the femur only showed a small shadow, indicating patchy bone sclerosis, in addition to thickening of the neighboring bone cortex and a pathological fracture. Another case showed inhomogeneous enhancement of the tumor.

MRI manifestations of osteosarcoma often consisted of low mixed signals on T1WI and high mixed signals on T2WI. The surrounding soft-tissue masses appeared as equally long T1and slightly long T2 mixed signals. The epiphysis and epiphyseal plate were invaded in 17 cases, and fat-suppressed T2WI provided the clearest images. Enhanced MRI (2/25) showed markedly inhomogeneous enhancement in the areas of bone destruction and the soft-tissue mass (Fig. 1). Bone scintigraphy $(19 / 25)$ showed abnormal radionuclide uptake by the lesions. PET-CT $(1 / 25)$ showed increased ${ }^{18} \mathrm{~F}-\mathrm{FDG}$ metabolism in the tibial bone marrow [standardized uptake value (SUV), 20.77]. A case assessed by ultrasonography showed a mixed-echoic soft-tissue mass adjacent to the humerus, and color Doppler flow imaging detected abundant blood flow signals.

MRI showed inhomogeneous bone signals of the lesions produced in the case of telangiectatic osteosarcoma, with low signals on T1WI and high mixed signals on T2WI. The surrounding soft tissues showed mixed signals and contained multiple cysts showing fluid-fluid levels (Fig. 2).

Ewing sarcoma. There were 5 cases of Ewing sarcoma ( 3 males and 2 females), with ages ranging from 4 to 17 years. The lesions were located in the right proximal femur of 3 cases, the sacral vertebrae of 1 case and the rib of 1 case. The size of the tumors ranged from $6.3-19.7 \mathrm{~cm}$. A pathological fracture was found in one case, whereas another case exhibited lung metastasis and multiple vertebral metastases.

Radiography (2/5) and CT (3/5) showed infiltrative bone destruction and soft-tissue masses. Lesions in the long bone showed a laminar periosteal reaction in 2 cases (Fig. 3), and the case with the pathological fracture showed a ruptured periosteum. Enhanced CT (1/5) showed marked inhomogeneous enhancement of the tumor, with enhanced vessels present within.

MRI (4/5) of tumors showed equal or slightly lower signal compared with adjacent normal bone marrow on T1WI and high mixed signal on T2WI. Enhanced MRI (1/5) showed markedly inhomogeneous enhancement. Bone scintigraphy (4/5) showed the increased radionuclide uptake of the lesions.

Chondrosarcoma. There were 3 cases of chondrosarcoma ( 2 males and 1 female), with ages ranging from 10 to 16 years. The lesions were located in the proximal tibia, distal femur and os pubis. The size of the tumors ranged from $4.9-10.1 \mathrm{~cm}$. Overall, 1 case was a central/intramedullary chondrosarcoma and 2 cases were periosteal/juxtacortical chondrosarcomas. The histopathology included 2 cases of mesenchymal chondrosarcoma and 1 case of grade 2 chondrosarcoma Chondrosarcoma are histologically differentiated into World Health Organization
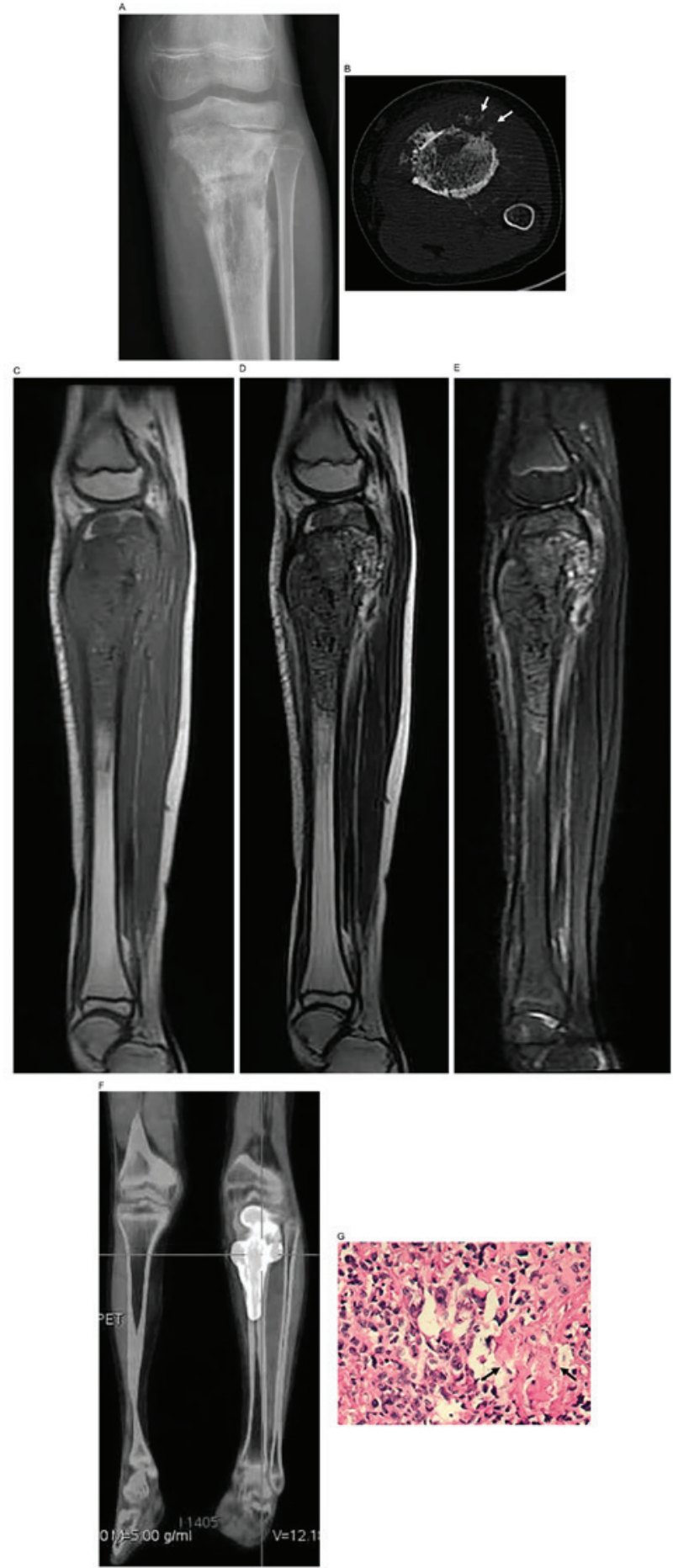

Figure 1. Osteosarcoma in a female patient aged 10 years. Osteosarcoma of left tibia. (A) Anteroposterior radiograph (B) and CT showing mixed osteosarcoma in the metaphysis of the proximal tibia, with radial periosteal reaction and a Codman triangle. Tumor osteoid matrix can be observed in the soft-tissue mass (arrows). (C) Sagittal T1WI, (D) T2WI and (E) fat-suppressed T2WI showing low T1WI/high T2WI mixed signals of the left tibia. The epiphysis is involved. $(\mathrm{F}){ }^{18} \mathrm{~F}$-fluorodeoxyglucose positron emission tomography-CT metabolic imaging showing increased radionuclide uptake (standardized uptake value, 20.77). (G) Photomicrograph showing patchy distribution of tumor cells, marked nuclear atypia, numerous mitotic figures and osteoid matrix (arrows) (original magnification, x200; hematoxylin and eosin staining). CT, computed tomography; WI, weighted images.

grade 1, 2 or 3, depending on cellularity, cellular atypia and mitosis (9). A single case had a pathological fracture. 

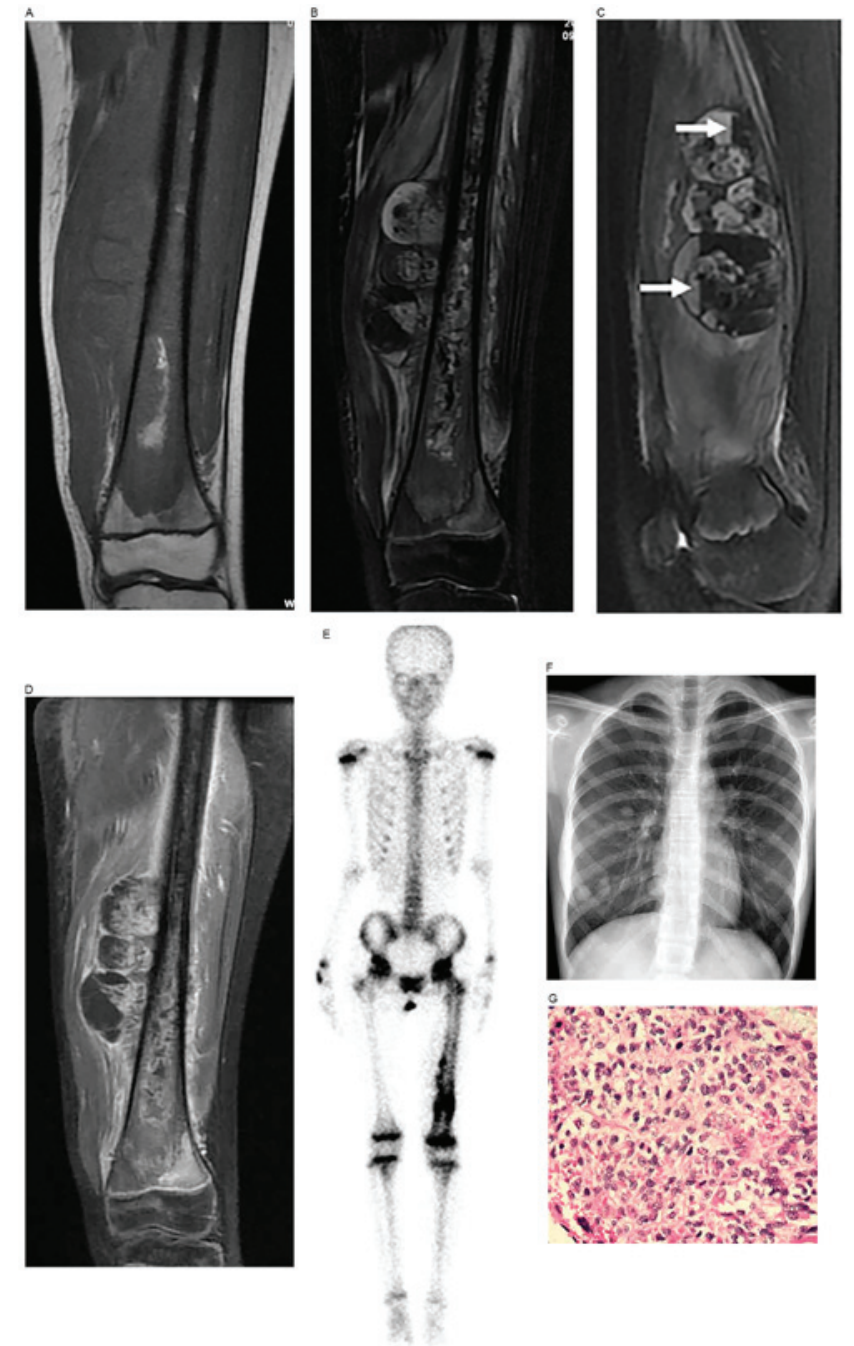

Figure 2. Telangiectatic osteosarcoma in a female patient aged 11 years Osteosarcoma of the left femur. (A) Coronal T1WI and (B) fat-suppressed T2WI showing inhomogeneous signals of bone in the area of the lesion, which were low signal on T1WI and high mixed signal on T2WI. The soft-tissue mass appears as a mixed signal and contains numerous cysts. (C) Sagittal fat-suppressed T2WI showing fluid-fluid levels in the cysts (arrows). (D) Coronal post contrast T1WI showing inhomogeneous enhancement of the area of bone destruction and the soft-tissue mass. (E) Whole-body technetium-99 m methylenediphosphonate bone scan showing inhomogeneous radionuclide uptake of the lower and middle sections of the left femur. (F) Anteroposterior chest radiograph showing numerous metastases in the lungs. (G) Photomicrograph showing marked nuclear atypia of tumor cells and numerous mitotic figures. The diagnosis was of telangiectatic osteosarcoma (original magnification, x200; hematoxylin and eosin staining). WI, weighted images.

The lesion of 1 case (periosteal/juxtacortical chondrosarcoma) was adjacent to the cortex of the proximal tibia, and was shown on radiography and $\mathrm{CT}$ as local destruction of the cortical bone of the lateral tibia, with a soft-tissue mass containing scattered punctate and amorphous calcifications. MRI showed long T1 W/long T2 W mixed signals. The pathological diagnosis was of mesenchymal chondrosarcoma (Fig. 4).

The lesion of another case (central/intramedullary chondrosarcoma) was located in the medullary cavity of the distal femur. Radiography showed a local periosteal reaction. MRI showed a low signal on T1WI and a high mixed signal on T2WI, with a periosteal reaction and soft-tissue mass. The epiphysis and epiphyseal plate were involved. The histopathological diagnosis was of mesenchymal chondrosarcoma.

Radiography and CT of 1 case showed local bone destruction of the right pubic ramus, with patchy punctate, cambered calcifications in the surrounding soft-tissue mass. MRI of the soft-tissue mass showed an equal signal on T1WI and a heterogeneous high signal on T2WI. The calcifications and septum of the mass showed low signals on T1WI and T2WI. The histopathological diagnosis was of grade II chondrosarcoma (Fig. 5).

Of the total cases, 2 underwent bone scintigraphy, which showed foci of increased radionuclide uptake.

Primary non-Hodgkin lymphoma of the bone. There was 1 case (male, 17 years old) of primary non-Hodgkin lymphoma of the bone involving the T12, L1 and L2 vertebrae. The tumor measured $2.7 \times 4.1 \times 6.5 \mathrm{~cm}$. No lymph nodes and internal organs were involved. CT showed infiltrative bone destruction of the vertebrae, a soft-tissue mass and a compression fracture of the L1 vertebra, without an evident periosteal reaction. MRI showed inhomogeneous shadowing with long-equal T1WI/long T2WI signals of the vertebrae. The spinal canal was narrowed, and the spinal cord was compressed due to localized kyphosis. The surrounding soft-tissue mass was shown as possessing equal T1WI/long T2WI signals. The areas of the T12, L1 and L2 vertebrae, and the soft-tissue mass showed marked inhomogeneous enhancement (Fig. 6). Bone scintigraphy showed slightly increased radionuclide uptake of the lesions. The pathological diagnosis was of non-Hodgkin lymphoma (anaplastic large cell lymphoma).

\section{Discussion}

In the present study, there were only a few more male patients than female patients, with an approximate ratio of $1: 1$. The age of onset of 27 of the 34 cases $(79 \%)$ was $<14$ years, indicating that the majority of the malignant bone tumors in the cohort occurred in children ( $<14$ years of age). The mean ages at onset of Ewing sarcoma, osteosarcoma and chondrosarcoma were 8, 11 and 13 years, respectively. Osteosarcoma was the most common primary malignant bone tumor (74\%), followed by Ewing sarcoma (15\%), chondrosarcoma (8\%) and lymphoma $(3 \%)$. The most common site of the osteosarcomas, Ewing sarcomas and chondrosarcomas was the long bones. Overall, $\sim 76 \%$ originated near the knee joint (distal femur and proximal tibia), which is consistent with the results of previous studies. The most common presenting symptom was pain, or an enlarging soft-tissue mass and pathological fractures. However, pathological fracture is one of the factors contributing to a poor prognosis and is indicative of aggressive tumors (10). The most common sites of metastasis were the lungs and other bones, while the lymph nodes and internal organs were rarely involved. Skip metastases have been reported in 2-6.5\% of osteosarcoma and 4-6\% of Ewing sarcoma patients $(6,11)$. Skip lesions occurred in $8 \%$ of the osteosarcoma patients in the present study, which is a slightly higher rate than that reported in the literature. None of the patients with Ewing sarcoma developed skip lesions.

The selection of imaging protocols for bone tumors in children should follow the 'As Low As Reasonably Achievable' 

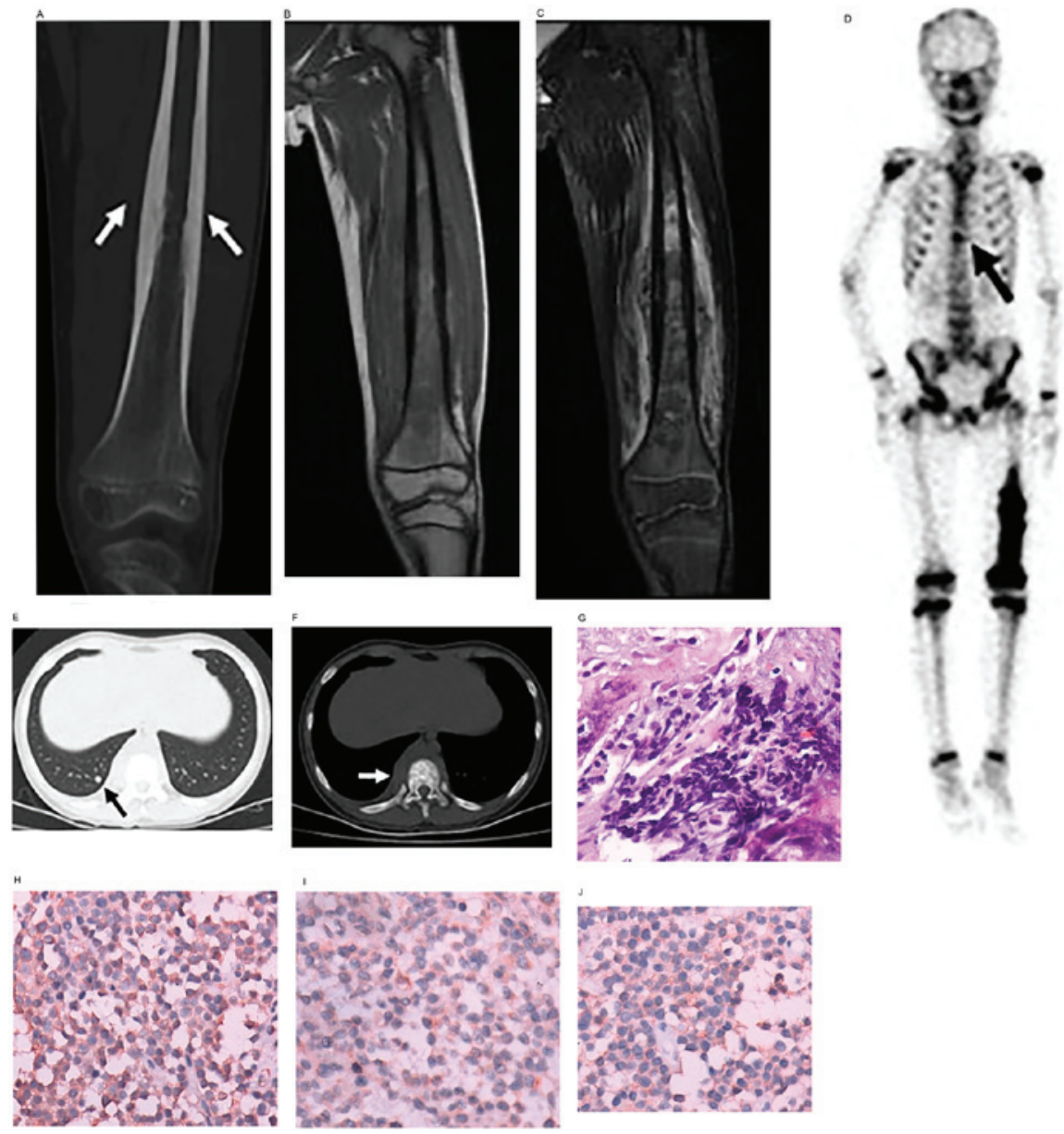

Figure 3. Ewing sarcoma in a male patient aged 11 years. Ewing sarcoma of the left femur. (A) Coronal reconstruction of CT showing laminar periosteal thickening of the femoral diaphysis (arrows), with osteoid matrix in the medullary cavity. (B) Coronal T1WI and (C) fat suppression T2WI showing inhomogeneous bone signals in the area of the lesion, with low signal and high mixed signal, and a soft-tissue mass with mixed signal, respectively. (D) Whole-body technetium-99 m methylenediphosphonate bone scan showing increased radionuclide uptake of the lower and middle sections of the left femur, and increased radionuclide uptake at the T10 vertebra (arrow), which indicates bone metastasis. Axial CT of the chest in (E) lung windows showing metastatic nodules in the lungs (arrow), and in (F) bone windows showing osteoblastic bone metastasis of the T10 vertebra, with surrounding soft-tissue swelling (arrow). (G) Photomicrograph showing uniform, small round tumor cells (original magnification, x400; hematoxylin and eosin staining). (H) Vimentin-positive, (I) cluster of differentiation 99-positive and (J) neuron-specific enolase-positive tissues (original magnification, $\mathrm{x} 400$; immunohistochemical staining). The diagnosis was of Ewing sarcoma. CT, computed tomography; WI, weighted images.

principle, which refers to minimizing the dose of ionizing radiation to the child, while ensuring image quality (6). Therefore, conventional radiography is the first choice, and in the majority of cases can identify benign and malignant tumors.

Bone scintigraphy is a method with high sensitivity, but low specificity. Due to osteogenetic activity or the increase in blood flow to benign and malignant bone tumors, the uptake of radionuclide in the lesions is increased. Therefore, in the majority of cases, Bone scintigraphy cannot differentiate benign from malignant tumors; however, it can be used to exclude benign tumors without radionuclide uptake. In addition, Bone scintigraphy is effective for detecting metastases of the skeletal system $(5,8)$. CT is more effective than conventional radiography at revealing subtle destruction of the bone cortex, the type of tumor matrix and periosteal reactions. Three-dimensional CT is the best option for bone tumors in the pelvis, scapula and other complex or overlapping bones. Additionally, CT is useful for finding pulmonary metastases $(5,8)$.
MRI is superior for evaluating the extent of intramedullary and soft-tissue masses. MRI is also markedly more efficient at detecting skip lesions $(5,8)$.

Functional MRI is able to differentiate not only benign and malignant bone tumors, but to a certain degree, to also depict the pathological processes of malignant bone tumors, including cell apoptosis, cell proliferation and angiogenesis $(2,12-14)$. Although functional MRI is in the research stage, the prospect for clinical applications is promising. At present, functional MRI techniques for the evaluation of bone tumors include the following: i) Quantitative dynamic contrast-enhanced (DCE) -MRI can be used to estimate the extent of necrosis in bone tumors. The percentage of tumor necrosis is the most important outcome measure for evaluating whether or not neoadjuvant therapy is clinically effective (1). Studies have shown that tumor necrosis of $<90 \%$ indicates a high risk of local recurrence and a short overall survival time $(6,15)$. The flux rate constant and dynamic vector magnitude (DVM) are commonly used quantitative parameters. A DVM of $>1.8$ indicates a poor response to treatment (necrosis $<90 \%$ ), while a DVW of $<1.8$ 

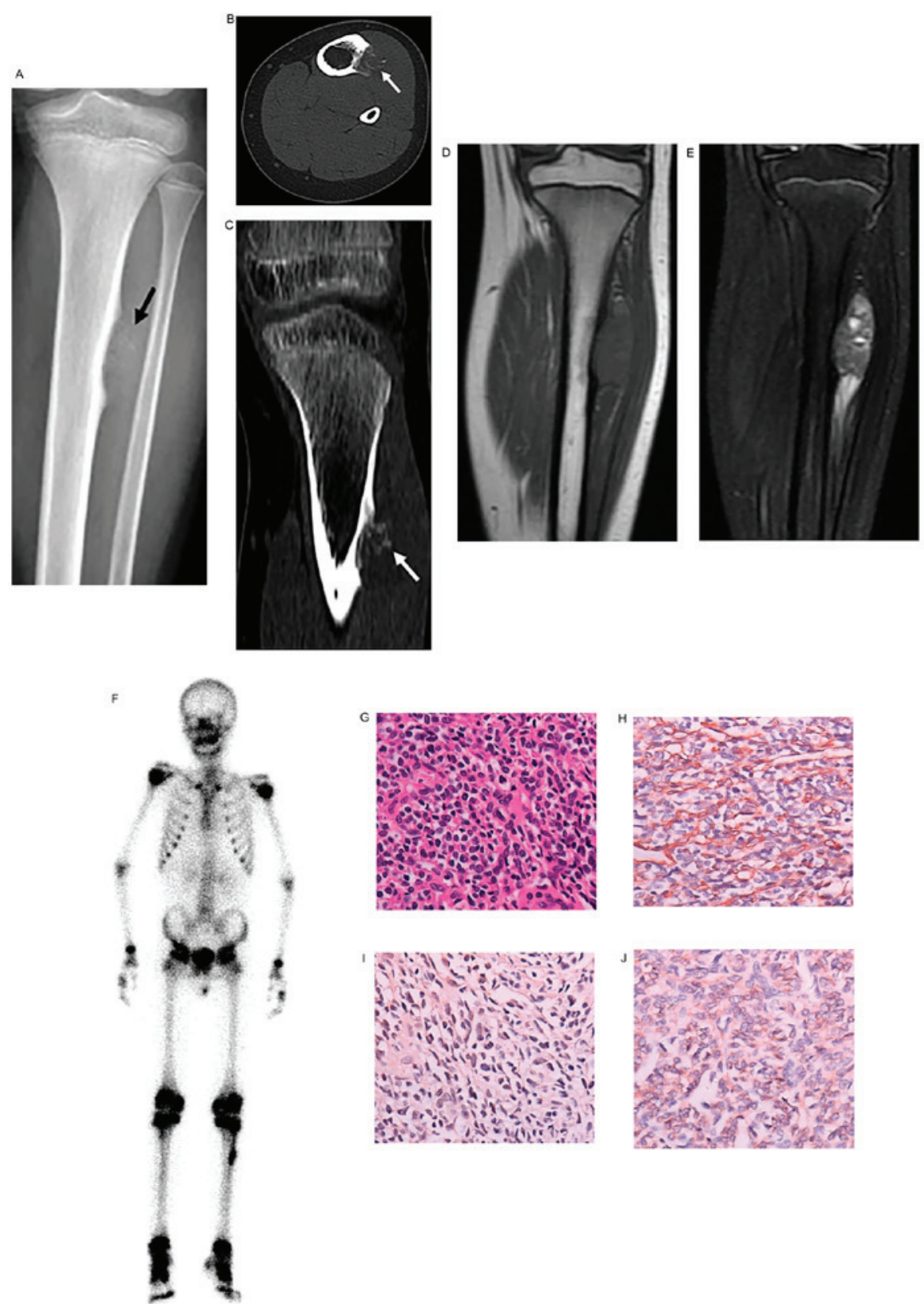

Figure 4. Periosteal/juxtacortical chondrosarcoma in a male aged 10 years. Chondrosarcoma of the left tibia. (A) Anteroposterior radiograph, (B) axial CT and (C) coronal reconstructed CT showing localized osteolytic destruction of the lateral bone cortex of the tibia, surrounded by a soft-tissue mass containing scattered calcifications of cartilage matrix (arrows). (D) Coronal T1WI and (E) fat-suppressed T2WI showing a lesion with equal signal and inhomogeneous high signal, respectively. (F) Whole-body technetium-99 m methylenediphosphonate bone scan showing increased radionuclide uptake of the upper section of the left tibia. (G) Photomicrograph showing small round tumor cells, with diffuse and intensive distribution (original magnification, $\mathrm{x} 400$; hematoxylin and eosin staining). (H) Vimentin-positive, (I) cluster of differentiation 99-positive and (J) S-100-positive tissues (original magnification, x400; immunohistochemical staining). The diagnosis was of mesenchymal chondrosarcoma. CT, computed tomography; WI, weighted images.

indicates a good response to treatment (necrosis >90\%) (1). ii) Diffusion-weighted (DW)-MRI is used to reflect the limitations of the diffusion of water in the body, in the presence of a pathological process. The apparent diffusion coefficient (ADC) is a quantitative index that reflects the apparent freedom of diffusion. Low ADC values indicate areas where diffusion is restricted by the intact cell membranes in tumor tissues, while high ADC values indicate acellular regions. Effective treatment can destroy the membranes of tumor cells, resulting in the acceleration of water diffusion $(12,13)$. A marked increase in the ADC value after treatment can be used as a marker for assessing tumor necrosis (1). Therefore DW-MRI can provide information about tissue cellularity and cell integrity, and is also effective at identifying benign and malignant tumors, and assessing response to treatment. Whole-body DW-MRI can be applied to the detection of primary tumors and bone metastases. iii) Magnetic resonance spectroscopy (MRS) is applied to quantify the levels of different metabolites, and thus depicts the molecular constitution of tumors. Studies have shown a marked increase in choline levels in malignant bone tumors. A choline/lipid ratio of $>0.2$ has been used to diagnose malignant bone tumors, with a sensitivity of $76 \%$ and a specificity of $88 \%$ (16). Therefore, by quantitative detection of changes in metabolite concentrations, this technique can 

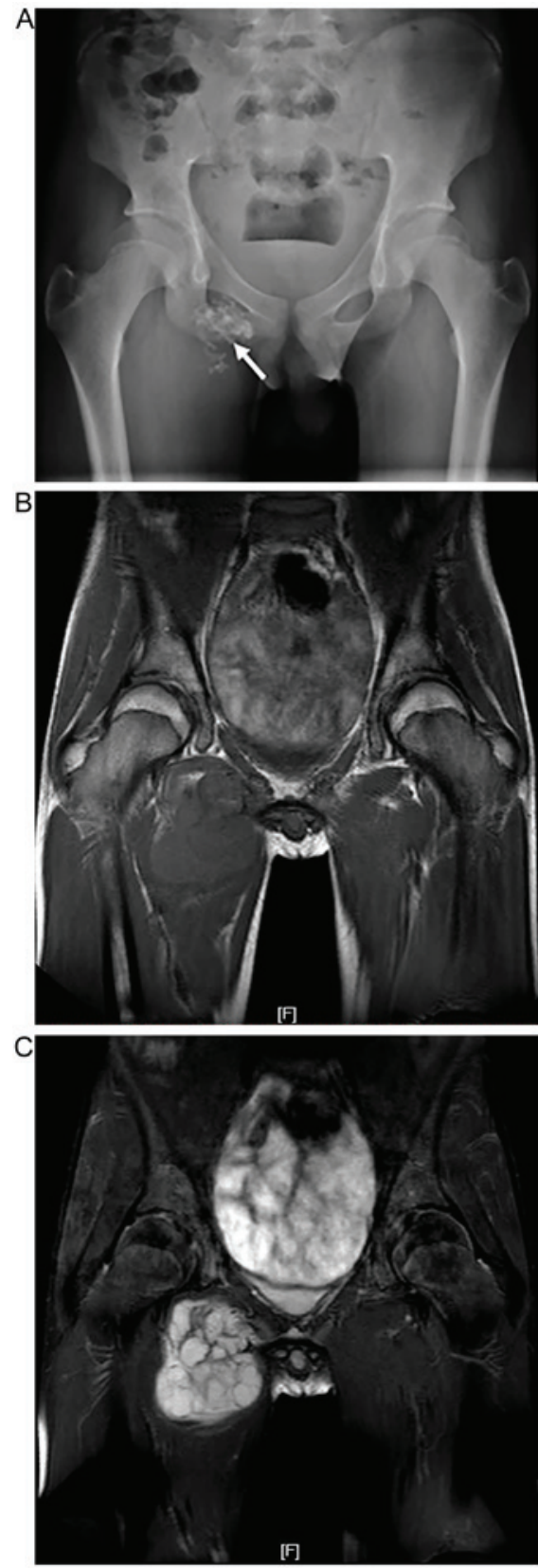

Figure 5. Chondrosarcoma in a male patient aged 16 years. Chondrosarcoma of the right pubis. (A) Anteroposterior radiograph of the pelvis showing bone destruction of the right pubic ramus, with punctate, patchy, circular and calcified cartilage matrix in the surrounding soft-tissue mass (arrow). (B) Corona T1WI and (C) fat-suppressed T2WI showing a soft-tissue mass with equal signal and inhomogeneous high signal, respectively, and ossification and a septum with low signal on T1WI and low signal on T2WI. WI, weighted images.

identify tumor recurrence and assess the response to treatment. iv) Blood-oxygen level-dependent imaging (BOLD) is another functional MRI technique, first developed for examining brain and now being used in other systems. The source of BOLD MRI signal indicates the ratio of oxyhaemoglobin and deoxygenated haemoglobin in the arterioles, capillaries and post-capillary venules, which is mainly used to assess oxygenation status in tumors. Oxygen concentration is a prognostic factor in recurrence of solid tumors, thus a BOLD sequence can be part of pre-therapy and follow-up MRI protocols in skeletal oncology $(17,18)$.
${ }^{18} \mathrm{~F}-\mathrm{FDG} \mathrm{PET} / \mathrm{CT}$ is most commonly used for the staging of malignant bone tumors; it plays important roles in detecting metastasis, assessing recurrence, guiding clinical surgery and radiotherapy, and assessing therapeutic efficacy $(5,8)$.

Osteosarcoma is the most common malignant bone tumor in children (6). In the present study, the majority of the lesions were located around the knee joint (distal femur and proximal tibia), and over $33 \%$ of cases involved the epiphysis, while $8 \%$ involved the diaphysis. These data are consistent with the literature (5).

The most common bone tumor on imaging is mixed osteosarcoma, which refers to the manifestation of osteolytic bone destruction and osteoblastic bone sclerosis. In the present study, mixed osteosarcoma accounted for $68 \%$ (17/25) of all osteosarcomas, the osteolytic type accounted for $16 \%(4 / 25)$ and the osteoblastic type accounted for $16 \%$ (4/25). Approximately $96 \%$ (24/25) showed the Codman triangle and radial, spiculate, patchy and other aggressive periosteal reactions. In total, 92\% (23/25) showed a soft-tissue mass, and 84\% (21/25) showed osteoid matrix in the areas of bone destruction and a soft-tissue mass. The 3 imaging features, aggressive periosteal reaction, soft-tissue mass and osteoid matrix are the most typical diagnostic signs for the diagnosis of malignant bone tumors.

Telangiectatic osteosarcoma is rare, accounting for $1.2-7 \%$ of all osteosarcomas. Approximately $90 \%$ of the lesions are located at the metaphysis of the long bones, and the femur $(50 \%)$ and tibia $(25 \%)$ are the most commonly affected bones (19). Dilated blood-containing cavities with fluid-fluid levels that are in the tumor are characteristic features, and resemble aneurysmal bone cysts. However, a thickened cyst wall and septum in a telangiectatic osteosarcoma can appear as a solid node on enhanced imaging (20).

Ewing sarcoma is the second most common malignant bone tumor in children (21). The onset age of Ewing sarcoma is younger than the onset age of osteosarcoma. In the present study, $80 \%$ of cases had an onset age of $<10$ years. The most common site of Ewing sarcoma is the pelvis, followed by the diaphysis of the long bones (femur, tibia, humerus and rib) (1). However, data from the present study revealed inconsistent results: The most common sites of involvement were the diaphysis of the long bones, then the pelvis. It was likely that this result was obtained because the number of patients was too small for adequate statistical analysis.

The imaging manifestations are moth-eaten, permeative bone destruction, a soft-tissue mass adjacent to the bone, and onion-skin or spiculate invasive periosteal reactions. The soft-tissue mass around the tumor is often larger than the area of bone destruction. The prognosis is poor when the size of the tumor is $>8 \mathrm{~cm}$. Calcification is rare in the areas of bone destruction and the soft-tissue mass $(6,19)$.

Chondrosarcoma is rare in children. The majority of these tumors are primary low-grade chondrosarcomas. As little as $0.5 \%$ of low-grade chondrosarcomas arise secondarily from benign chondroid lesions (22). Chondrosarcomas are most commonly located in the femur, pelvis or shoulder, and originate from the proximal femur in one-third of cases. Approximately 50\% are located at the metaphysis, 35\% at the diaphysis and $15 \%$ at the epiphysis (22).

The size of chondrosarcoma is large, normally $>4 \mathrm{~cm}$, or even up to $10-15 \mathrm{~cm}$. In the majority of cases, conventional 

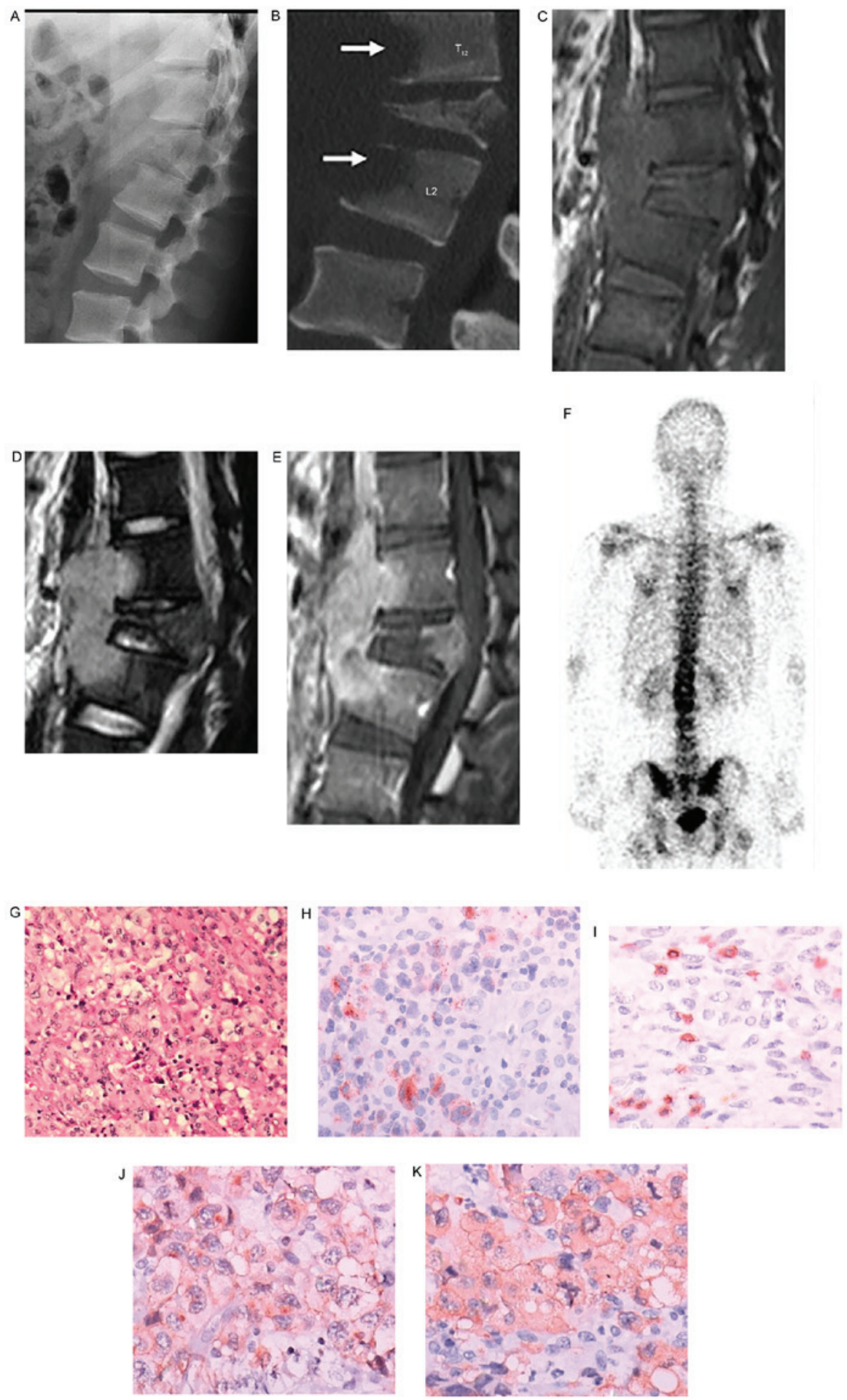

Figure 6. Primary bone lymphoma in a male patient aged 17 years. Non-Hodgkin lymphoma of the vertebra. (A) Lateral view radiograph and (B) sagittal reconstructed CT showing a compression fracture of the L1 vertebra, and anterior bone destruction of the T12 and L2 vertebrae (arrows). (C) Sagittal T1WI and (D) T2WI showing bone destruction with a low signal on T1WI and a slightly high signal on T2WI. A soft-tissue mass is observed adjacent to the vertebrae, without evident narrowing of the intervertebral space. (E) Post-contrast T1WI with fat suppression showing marked enhancement of the area of vertebral destruction and the mass. The findings were misdiagnosed as lumbar vertebral tuberculosis prior to surgery. (F) Whole-body technetium-99 m methylenediphosphonate bone scan showing slightly increased radionuclide uptake of the L1 and L2 vertebrae. (G) Photomicrograph showing diffuse heterocysts or nests of heterocysts, with inhomogeneous sizes and marked atypia (original magnification, x200; hematoxylin and eosin stain). (H) Epithelial membrane antigen-positive, (I) CD3-positive, (J) CD30-positive and (K) anaplastic lymphoma kinase-positive (original magnification, x400; immunohistochemical stain). Non-Hodgkin lymphoma (anaplastic large cell lymphoma) was diagnosed. CD, cluster of differentiation; WI, weighted images.

radiography reveals osteolytic bone destruction and a punctate, patchy, circular and cambered cartilaginous matrix. However, differentiating a low-grade chondrosarcoma from enchondroma is difficult. The following are the key identifying features $(8,22)$ : i) The depth of endosteal scalloping is more than two-thirds of the thickness of the bone cortex on radiography or CT. ii) The extent of edema around the chondrosarcoma is often larger than that of an enchondroma 
on MRI. iii) A chondrosarcoma shows peripheral, nodular or septal enhancement on contrast-enhanced CT or MRI, whereas an enchondroma only shows peripheral enhancement. DCE-MRI reveals a fast enhancement rate for chondrosarcoma. iv) ${ }^{18}$ FDG PET-CT SUVs of $>2.0$ have a $91 \%$ sensitivity, $100 \%$ specificity and $95 \%$ accuracy for identifying chondrosarcoma. v) Whole-body ${ }^{99 \mathrm{~m}} \mathrm{Tc}-\mathrm{MDP}$ bone scans show higher radionuclide uptake in a chondrosarcoma than the uptake in the normal iliac crest.

Mesenchymal chondrosarcoma is a rare tumor, accounting for only 3-10\% of all chondrosarcomas, and is highly malignant (6). Approximately $70 \%$ of mesenchymal chondrosarcomas occur in young adults aged 20 to 30 years (23). The 2 cases of mesenchymal chondrosarcoma in the present study showed bone destruction and a punctate, circular cartilaginous matrix, with no characteristic imaging features. Therefore histopathological examination was required for a definitive diagnosis. Histologically, this biphasic tumor of the cartilage is composed of hyaline cartilage and a small round cell component, and is extremely rare (24).

Primary bone lymphoma accounts for $<5 \%$ of all primary bone tumors, and is more common in males, with a male/female ratio of 1.5:1. The majority of the cases are non-Hodgkin lymphoma (25). Primary bone lymphoma in children is likely to occur in the spine or the diaphyses of the long bones. The following are the diagnostic criteria of primary bone lymphoma proposed by the World Health Organization (26): i) Single-bone invasion, with/without local lymph node involvement; and ii) multiple-bone invasion, without involvement of the lymph nodes and internal organs. CT of the chest, abdomen and pelvis must be performed to exclude lymph node metastasis, which indicates stage IV disease.

The present study contained 1 patient with primary bone lymphoma with multiple bone involvement, which had been misdiagnosed as vertebral tuberculosis preoperatively. According to the literature, primary bone lymphoma with multiple-bone involvement is prone to occur in the vertebrae, as opposed to primary bone lymphoma with single-bone involvement. Primary bone lymphoma can infiltrate tissues through the vascular channels of the bone cortex; therefore, the bone cortex may not be damaged during the formation of a soft-tissue mass $(1,8)$, and there also may not be a marked periosteal reaction. Calcifications are rare, and sequestra can be observed in a few cases (19).

In general, children cannot provide a clear medical history of their complaints; therefore, useful diagnostic information may be difficult to obtain. In addition, children may not discover the problem early in the course of the disease, and parents may not observe the signs of disease. Therefore, children are examined by a physician when the lesions are large, or when they have symptoms caused by pressure or metastatic disease, which negatively affects the chances for early diagnosis and treatment, and the resultant prognosis. At present, there are few pediatric oncologists in China (27), and lay people have limited awareness of pediatric tumors. Therefore, children with primary malignant bone tumors often do not receive timely and effective treatment.

In conclusion, in the present study cohort and in general, osteosarcoma is the most common primary malignant bone tumor in children, followed by Ewing sarcoma, chondrosarcoma and lymphoma. Osteosarcoma is more frequently found in children $<14$ years old, and is most commonly located in the long tubular bones of the lower extremities. Osteoblastic or osteolytic bone destruction, an invasive periosteal reaction, a soft-tissue mass, the tumor matrix and inhomogeneous enhancement are important imaging features of malignant bone tumors. Conventional radiography is the preferred method of examination, and CT can be used as a supplementary method. MRI is used to determine the extent of tumor invasion, and combined with functional MRI and ${ }^{18} \mathrm{~F}-\mathrm{FDG}$ PET/CT, can be used for preoperative evaluation and treatment assessment.

\section{References}

1. Wootton-Gorges SL: MR Imaging of primary bone tumors and tumor-like conditions in children. Magn Reson Imaging Clin N Am 17: 469-487, 2009.

2. Brisse H, Ollivier L, Edeline V, Pacquement H, Michon J, Glorion $\mathrm{C}$ and Neuenschwander S: Imaging of malignant tumours of the long bones in children: Monitoring response to neoadjuvant chemotherapy and preoperative assessment. Pediatr Radiol 34: 595-605, 2004.

3. Collins M, Wilhelm M, Conyers R, Herschtal A, Whelan J, Bielack S, Kager L, Kühne T, Sydes M, Gelderblom H, et al: Benefits and adverse events in younger versus older patients receiving neoadjuvant chemotherapy for osteosarcoma: Findings from a meta-analysis. J Clin Oncol 31: 2303-2312, 2013.

4. Wright EH, Gwilym S, Gibbons CL, Critchley P and Giele HP: Functional and oncological outcomes after limb-salvage surgery for primary sarcomas of the upper limb. J Plast Reconstr Aesthet Surg 61: 382-387, 2008.

5. Eftekhari F: Imaging assessment of osteosarcoma in childhood and adolescence: Diagnosis, staging and evaluating response to chemotherapy. Cancer Treat Res 152: 33-62, 2009.

6. Kaste SC: Imaging pediatric bone sarcomas. Radiol Clin North Am 49: 749-765, 2011.

7. Costelloe CM and Madewell JE: Radiography in the initial diagnosis of primary bone tumors. AJR Am J Roentgenol 200: 3-7, 2013.

8. Rana KA, Meyer J, Ibrahim S, Ralls M and Kent PM: The role of imaging of malignant bone tumors in children and young adults. Curr Probl Cancer 37: 181-191, 2013.

9. Fletcher CDM, Bridge JA, Hogendoorn PCW and Mertens F: World Health Organization classification of tumours of soft tissue and bone [M]. Lyon, IARC Press, pp264-81, 2013.

10. Lee RK, Chu WC, Leung JH, Cheng FW and Li CK: Pathological fracture as the presenting feature in pediatric osteosarcoma. Pediatr Blood Cancer 60: 1118-1121, 2013.

11. Murphey MD, Senchak LT, Mambalam PK, Logie CI, Klassen-Fischer MK and Kransdorf MJ: From the radiologic pathology archives: Ewing sarcoma family of tumors: Radiologic-pathologic correlation. Radiographics 33: 803-831, 2013.

12. Fayad LM, Jacobs MA, Wang X, Carrino JA and Bluemke DA: Musculoskeletal tumors: How to use anatomic, functional and metabolic MR techniques. Radiology 265: 340-356, 2012.

13. Benassi MS, Rimondi E, Balladelli A, Ghinelli C, Magagnoli G and Vanel D: The role of imaging for translational research in bone tumors. Eur J Radiol 82: 2115-2123, 2013.

14. Hwang S and Panicek DM: The evolution of musculoskeletal tumor imaging. Radiol Clin North Am 47: 435-453, 2009.

15. Meyers PA, Gorlick R, Heller G, Casper E, Lane J, Huvos AG and Healey JH: Intensification of preoperative chemotherapy for osteogenic sarcoma: Results of the Memorial Sloan-Kettering (T12) protocol. J Clin Oncol 16: 2452-2458, 1998.

16. Zhang J, Cheng K, Ding Y, Liang W, Ding Y, Vanel D and Cheng X: Study of single voxel $1 \mathrm{H}$ MR spectroscopy of bone tumors: Differentiation of benign from malignant tumors. Eur J Radiol 82: 2124-2128, 2013.

17. Dallaudiere B, Hummel V, Hess A, Lincot J, Preux PM, Maubon A and Monteil J: Tumoral hypoxia in osteosarcoma in rats: Preliminary study of blood oxygenation level-dependent functional MRI and 18F-misonidazole PET/CT with diffusion-weighted MRI correlation. AJR Am J Roentgenol 200: 187-192, 2013. 
18. Padhani AR, Krohn KA, Lewis JS and Alber M: Imaging oxygenation of human tumours. Eur Radiol 17: 861-872, 2007.

19. Yarmish G, Klein MJ, Landa J, Lefkowitz RA and Hwang S: Imaging characteristics of primary osteosarcoma: Nonconventional subtypes. Radiographics 30: 1653-1672, 2010.

20. Murphey MD, wan Jaovisidha S, Temple HT, Gannon FH, Jelinek JS and Malawer MM: Telangiectatic osteosarcoma: Radiologic-pathologic comparison. Radiology 229: 545-553, 2003.

21. Balamuth NJ and Womer RB: Ewing's sarcoma. Lancet Oncol 11: 184-192, 2010.

22. Mosier SM, Patel T, Strenge K and Mosier AD: Chondrosarcoma in childhood: The radiologic and clinical conundrum. J Radiol Case Rep 6: 32-42, 2012.
23. Küpeli S, Varan A, Gedikoğlu G and Büyükpamukçu M: Sacral mesenchymal chondrosarcoma in childhood: A case report and review of the literature. Pediatr Hematol Oncol 27: 564-573, 2010.

24. Qasem SA and DeYoung BR: Cartilage-forming tumors. Semin Diagn Pathol 31: 10-20, 2014.

25. Carroll G, Breidahl W and Robbins P: Musculoskeletal lymphoma: MRI of bone or soft tissue presentations. J Med Imaging Radiat Oncol 57: 663-673, 2013.

26. Demircay E, Hornicek FJ Jr, Mankin HJ and Degroot H III: Malignant lymphoma of bone: A review of 119 patients. Clin Orthop Relat Res 471: 2684-2690, 2013.

27. Hu KJ, Sun ZZ, Rui YJ, Mi JY and Ren MX: Shortage of paediatricians in China. Lancet 383: 954, 2014. 